\title{
PENETRATION OF INTERNAL GRAVITY WAVEGUIDE MODES INTO THE UPPER ATMOSPHERE
}

\section{G.V. Rudenko, I.S. Dmitrienko}

Institute of Solar-Terrestrial Physics SB RAS, Irkutsk, Russia

rud@iszf.irk.ru

The paper describes internal gravity waveguide modes, using dissipative solutions above the source. We compare such a description with an accurate approach and a WKB approximation for dissipationless equations. For waveguide disturbances, dispersion relations calculated by any method are shown to be close to each other and to be in good agreement with observed characteristics of traveling ionospheric disturbances. Unlike other methods, dissipative solutions above the source allow us to adequately describe the spatial structure of disturbances in the upper atmosphere.

Keywords: internal gravity waves, waveguide propagation, traveling ionospheric disturbances.

\section{INTRODUCTION}

This paper studies characteristics of trapped atmospheric modes extending over large heights. Such modes can be described by a dissipative solution above a source (DSAS) [Rudenko, Dmitrienko, 2015]. In fact, DSAS satisfies the upper boundary condition for trapped modes - lack of energy from above; and, since there is no source in the problem of trapped modes, DSAS is valid throughout the atmosphere. DSAS can actually take arbitrary values on Earth's surface. Thus, the problem of finding solutions for trapped modes reduces to the problem of selecting DSAS that fulfills the lower boundary condition for trapped modes - zero vertical velocity on Earth's surface; this boundary condition defines a dispersion equation for trapped modes.

We deal with internal gravity wave (IGW) modes of a waveguide formed by temperature stratification of the lower atmosphere. Such modes even without atmospheric dissipation cannot be described by solutions with real eigenvalues owing to sub-barrier penetration from the waveguide. Strictly speaking, the trapped modes of interest are collective rather than individual discrete modes. However, for our problem this circumstance is significant only because we should frame the solution above the source with complex frequency and wavenumber as opposed to [Rudenko, Dmitrienko, 2015] where calculations were based on real frequency and wavenumber parameters. In this study, we assume the real frequency as specified and find its respective complex horizontal wavenumber at which DSAS meets the lower boundary condition. Such DSAS is the waveguide mode. According to [Rudenko, 
Dmitrienko, 2015], DSAS results from a combination of three solutions: an analytical solution for the upper atmosphere $\left(R^{\mathrm{I}}\right)$, considered in an isothermal approximation, and numerical solutions for a real, nonisothermal dissipative atmosphere in the middle part $\left(R^{\mathrm{II}}\right)$ and for a real, nonisothermal one in a low dissipation approximation in the lower part $\left(R^{\mathrm{III}}\right)$. Thus, the solutions obtained for the waveguide modes relate their amplitude and other parameters in the lower atmosphere to their parameters in the upper isothermal atmosphere.

Describing amplitude characteristics of waveguide modes at large heights is extremely important, first for their experimental detection. While energy of the waveguide modes is largely concentrated at low heights in the region of their trapping, due to exponential increase in relative disturbance values, which is caused by atmospheric density decrease, we can observe them only indirectly, predominantly in the upper atmosphere. In this region, relative disturbance values are larger than those in the lower atmosphere; at those heights where disturbances are not completely suppressed by dissipation, they can generate considerable disturbances of a charged ionospheric component. Just due to the "invisible" IGW propagation, in the lower atmosphere we can observe a very common phenomenon of traveling ionospheric disturbances (TIDs).

The paper has the following structure. Section 1 describes the model atmosphere we employ for calculations. Section 2 deals with constructing and analyzing waveguide modes of IGW spectral range. We compare waveguide solutions found with DSAS to dissipationless approximation solutions obtained both with the WKB method and with numerical methods. Such comparisons pursue two goals at a time. First, they provide an insight into the dissipation effect on the main characteristics of waveguide propagation: dispersion relations, waveguide penetration and horizontal attenuation of waveguide modes. Second, they are additional tests to those performed in [Rudenko, Dmitrienko, 2015] both for a method of finding DSAS and for corresponding codes. We obtain dispersion characteristics and detailed description of the height structure of all disturbance components. The resulting waveguide solution has all characteristic features of trapped IGW in the real atmosphere: localization in view of temperature stratification; penetration through an opacity area; qualitative changes in the wave structure caused by dissipative disturbance propagation in the upper atmosphere. We receive full information about the entire height structure of waveguide modes that can be directly utilized to determine quantitative correlation between IGW modes and TIDs. We show that our waveguide solutions agree well with the main characteristics of TIDs following from observations: relations between periods and spatial scales, horizontal attenuation, values of total phase propagation velocity, slopes of phase fronts.

Notice that waveguide modes have been studied before [Francis, 1973a, b], and the results are being widely used for theoretical works and for interpreting observation of various disturbances, including those in the upper atmosphere [Shibata, Okuzawa, 1983; Afraimovich et al., 2001; Vadas, Liu, 2009; Vadas, Nicolls, 2012; Idrus et al., 2013; Heale et al., 2014; Hedlin, Drob, 2014 et al.]. Francis [Francis, 1973a, b] defined dispersion characteristics and vertical structures of waveguide modes. The author established that one or two lower IGW modes could exist at ionospheric heights due to waveguide penetration. Francis's 
method can calculate well enough the structure of wave disturbances in the lower atmosphere and dispersion characteristics of modes trapped by irregularities of the lower atmosphere. The method in terms of its applicability to the upper atmosphere is discussed in detail in [Rudenko, Dmitrienko, 2015]. Here we note only that its peculiarities, such as the common usage of reduction of order of differential equations admissible only in weak dissipation, cannot actually give a correct description of disturbances in the upper atmosphere. Unlike Francis's method, our method for finding DSAS employs reduction of order of wave equations to the second one (in our own way) only for heights of low dissipation where it makes sense. Thus, our method provides an adequate description for upper atmospheric disturbances.

\section{MODEL ATMOSPHERE}

Our calculations are based on a model atmosphere specified by the vertical profile of undisturbed temperature $T_{0}(z)$ according to distribution of NRLMSISE-2000 with the geographic coordinates of Irkutsk for the local midwinter noon:

$$
\begin{aligned}
& p_{0}(z)=p_{0}(0) e^{-\frac{g}{R} \int_{0}^{z} \frac{1}{T_{0}\left(z^{\prime}\right)} d z^{\prime}}, p_{0}(0)=1.01 \mathrm{~Pa} ; \\
& p_{0}(z)=p_{0}(0) e^{-\frac{g}{R} \int_{0}^{z} \frac{1}{T_{0}\left(z^{\prime}\right)} d z^{\prime}}, p_{0}(0)=1.01 \mathrm{~Pa}
\end{aligned}
$$

Here $p_{0}, \rho_{0}$ are undisturbed pressure and density; $z$ is a vertical coordinate measured from Earth's surface; $g=9.807 \mathrm{~m} / \mathrm{s}^{2}$ is the free fall acceleration; $R=287 \mathrm{~J} /(\mathrm{kg} \cdot \mathrm{K})$ is the universal gas constant. We consider that the atmosphere displays thermal conductivity, taking its dynamic coefficient as constant.

Since our method for finding wave solutions in addition to distribution of undisturbed temperature requires its first, second, and third derivatives (see [Rudenko, Dmitrienko, 2015]), as a calculation model we utilize an approximation of the model NRMSIS with smoothness up to the third derivative:

$$
\begin{aligned}
& T(z>430)=944.4 \\
& T(95.3<z \leq 430)=\left(\left[\cos \left(\frac{\pi}{2}\left(\frac{430-z}{430-95.3}\right)^{6}\right)\right]^{3}-1\right) \times(944.4-185.4)+944.4 \\
& T(46<z \leq 95.3)=-\left(\left[\cos \left(\frac{\pi}{2}\left(\frac{95.3-z}{95.3-46}\right)^{2}\right)\right]^{3}-1\right) \times(257-185.4)+185.4 \\
& T(20<z \leq 46)=\left(\left[\cos \left(\frac{\pi}{2}\left(\frac{z-20}{46-20}\right)^{2}\right)\right]^{3}-1\right) \times(215.1-257)=215.1 \\
& T(0<z \leq 20)=\left(\left[\cos \left(\arccos \left(0.5^{3 / 2}\right)\left(\frac{20-z}{20}\right)^{2}\right]^{3}-1\right) \times(215.1-270.1)+215.1 .\right.
\end{aligned}
$$

The initial dependence $T_{0}(z)$ and its approximation are given in Figure 1.

When selecting a model for winter, we focused on the fact that our results can be compared to the greatest extent with available particular observations for our region during the most reliable reception of 
TIDs. In summer, they are scarcely observed in our region. There may be two reasons for this: either disturbance sources do not reveal themselves or during this period waveguide cutoff conditions in assumed propagation paths are violated. Extensive discussion of these factors calls for a separate analysis of possible waveguide propagation paths at diverse locations of sources and receiving points.

\section{IGW WAVEGUIDE}

In this section, we examine low-frequency IGW disturbances occurring at ionospheric heights far from their sources. Since such waves cannot be trapped in the upper atmosphere (approximately isothermal), we can interpret them only as disturbances leaking from the IGW waveguide located at lower heights.

Next, we compare three methods for solving the waveguide problem: 1) dissipationless WKB description; 2) dissipationless numerical solution of boundary value problem; 3) waveguide solution with dissipation [Rudenko, Dmitrienko, 2015]. First, we give necessary formulas for each of the methods.

\subsection{Waveguide equations}

\section{Dissipationless WKB description}

Combined equations (7) [Rudenko, Dmitrienko, 2015] in the main order of WKB approximation readily yields a square of wavenumber as

$$
\left(k_{z}^{\mathrm{WKB}}\right)^{2}=U(z),
$$

where

$$
U(z)=-\frac{1}{4} \frac{\gamma^{2} g^{2}}{c_{s}^{4}}+(\gamma-1) \frac{k^{2} g^{2}}{c_{s}^{2} \omega^{2}}+\frac{\omega^{2}}{c_{s}^{2}}-k_{x}^{2} .
$$

In this paper, we adopt the same designations as in [Rudenko, Dmitrienko, 2015].

Discuss the profile of function $U$ (Figure 2) for arbitrarily chosen wave parameters $\omega$ and $k_{x}\left(T_{W}=2 \pi / \omega\right)=90 \mathrm{~min}, \lambda_{\text {hor }}=2 \pi / k_{x}=1390 \mathrm{~km}$. It is evident that the waveguide can be located in the region of propagation below the height $z_{1}$ due to the upper opacity region formed by negative values of $U$ in $z_{1}<z<z_{2}$. Above $z_{2}$ is the region of free vertical propagation into which a proportion of waveguide mode energy can penetrate. Note that a characteristic feature of the problem is a strong variation in shape and values of $U$ depending on the wave parameters $\omega$ and $k_{x}$. The height values $z_{1}$ and $z_{2}$ also vary.

We can show that in the case of sub-barrier penetration the waveguide cutoff condition with the penetration in the WKB approximation can be represented by a modified Bohr-Sommerfeld condition of quantization (MBSCQ) with complex turning points 


$$
\int_{C} \sqrt{U} d z \approx \pi\left(\frac{1}{2}+n\right)+i \exp \left[-2 \int_{z_{1}}^{z_{2}} \sqrt{U_{0}(z)} d z\right] \equiv \pi\left(\frac{1}{2}+n\right)+i Q, n=0,1, \ldots
$$

Condition (3) gives dispersion relations between real frequencies $\omega$ and the complex wavenumbers $k_{x}$ with a small imaginary part reflecting the degree of horizontal attenuation of the waveguide mode $n$. The contour of integration $C$ of the integral on the left side of Equality (3) starts at zero height $z_{0}$ and ends at a complex turning point $z_{c 1}$, close to the real turning point $z_{1}\left(U\left(z_{1}, \omega, \operatorname{Re} k_{x}\right)\right)$. In addition, if there are internal (complex) turning points, we assume that the contour $C$ also passes through these points (for simplicity, in our calculations we ignore contour pieces $C$ with $\operatorname{Re} U(z)<0)$. The integral in the argument of exponent on the right side of (3) is set real; index 0 of $U$ in the expression under integral sign implies that it is a function of the real part of $k_{x}$ and the real $z: U_{0}(z)=U\left(z, \omega, \operatorname{Re} k_{x}\right)$. Equation (3) is solved by the perturbation method. From equation

$$
\int_{0}^{z_{1}} \sqrt{\left|U_{0}(z)\right|} d z-\pi\left(\frac{1}{2}+n\right)=0
$$

for the chosen real $\omega$ we find a real root $k_{0 x}$ in Equation (4). Next, we introduce designations

$$
\begin{aligned}
& \int_{C} \sqrt{U} d z \approx \pi\left(\frac{1}{2}+n\right)+i \exp \left[-2 \int_{z_{1}}^{z_{2}} \sqrt{U_{0(z)}} d z\right] \equiv \pi\left(\frac{1}{2}+n\right)+i Q, n=0,1, \ldots, \\
& k_{x}=k_{0 x}(1+i \delta), U(z)=U_{0}(z)+k_{0 x} i \delta \frac{\partial}{\partial k_{x}} U_{0} .
\end{aligned}
$$

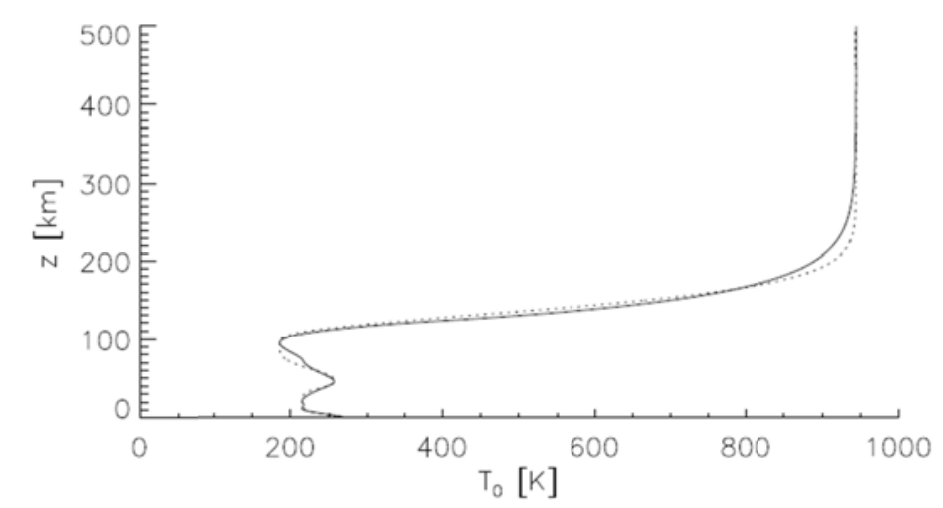

Figure 1. Initial temperature distribution (solid line); approximation (dotted line)

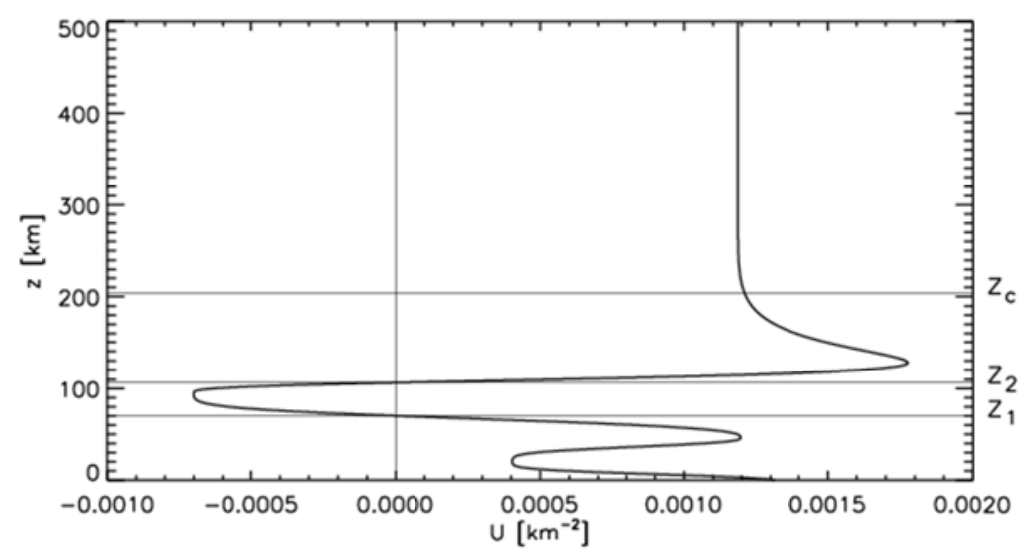

Figure 2. Characteristic vertical distribution of $U$ 
By substituting (5) in (3) and taking into account the complexity of the turning points $z_{c j}$ of the integration contour of integral on the left side of (3), derive an equation for complex addition to horizontal wave vector:

$$
\delta \frac{1}{2} \int_{0}^{z_{1}} \frac{k_{0 x}}{\sqrt{U_{0}}}\left(\frac{\partial}{\partial k_{x}} U_{0}\right) d z+\delta^{3 / 2} \frac{2}{3} e^{3 i \pi / 4} k_{0 x}^{3 / 2} \sum_{j}\left[\frac{\left(\partial U_{0} / \partial k_{x}\right)^{3 / 2}}{\left|\partial U_{0} / \partial z\right|}\right]_{z=z_{j}}=Q
$$

Equation (6) for $\delta^{1 / 2}$ has three roots from which we choose one corresponding to the waveguide mode attenuation.

\section{Dissipationless boundary value problem}

We can most conveniently formulate the boundary value problem (BVP), using a second-order differential equation for disturbed vertical velocity $v_{z}$. Such an equation can easily be derived from combined equations (2), (9), (10) from [Rudenko, Dmitrienko, 2015]

$$
\begin{aligned}
& p^{\prime}=a_{11} p+a_{12} v_{z}, \\
& v_{z}^{\prime}=a_{21} p+a_{22} v_{z},
\end{aligned}
$$

where

$$
\begin{aligned}
& a_{11}=\frac{p_{0}^{\prime}}{\gamma p_{0}} ; a_{12}=i \omega \rho_{0}\left(1-\frac{\omega_{N}^{2}}{\omega^{2}}\right), \\
& a_{21}=-\frac{i \omega}{\rho_{0}}\left(\frac{p_{0}^{\prime}}{\gamma p_{0} g}+\frac{k_{x}^{2}}{\omega^{2}}\right) ; a_{22}=-\frac{p_{0}^{\prime}}{\gamma p_{0}} .
\end{aligned}
$$

Differentiate the second equation from (7):

$$
v_{z}^{\prime \prime}=a_{21} p^{\prime}+a_{22} v_{z}^{\prime}+a_{21}^{\prime} p+a_{22}^{\prime} v_{z} \text {. }
$$

Then, employing the first equation from (7) for $p^{\prime}$ and expressing $p$ from the second equation from (7):

$$
\begin{aligned}
& p=\frac{1}{a_{21}}\left(v_{z}^{\prime}-a_{22} v_{z}\right), \text { obtain an equation for } v_{z}: \\
& v_{z}^{\prime \prime}+P v_{z}^{\prime}+B=0, \\
& P=-a_{11}-a_{22}-\left(\ln a_{21}\right)^{\prime}=\frac{T_{0}^{\prime}}{T_{0}\left(\frac{\omega^{2}}{k^{2} c_{s}^{2}}-1\right)}-\frac{\gamma g}{c_{s}^{2}}, \\
& B=-a_{21} a_{12}+a_{11} a_{22}+a_{22}\left(\ln a_{21}\right)^{\prime}-a_{22}^{\prime}= \\
& =-\frac{g}{c_{s}^{2}} P-\left(\frac{g}{c_{s}^{2}}-\frac{T_{0}^{\prime}}{T_{0}}\right) \frac{g}{c_{s}^{2}}+\frac{g}{c_{s}^{2}}\left(\frac{\omega^{2}}{c_{s}^{2}} k^{2}\right)\left(1-\frac{\omega_{N}^{2}}{\omega^{2}}\right) .
\end{aligned}
$$

It is convenient to solve BVP for Equation (9) by the respective nonlinear Riccati equation:

$$
G^{\prime}-P G-B G^{2}-1=0
$$


where $G$ is related to $v_{z}$ by

$$
G v_{z}^{\prime}=v_{z} \text {. }
$$

The waveguide solution function $G$ should satisfy the upper and lower boundary conditions. Given the upper boundary condition $\left(z=z_{\infty \rightarrow+\infty}\right), G$ should correspond to an upward propagating wave:

$$
G\left(z_{\infty}\right)=-\left(\frac{1}{2} P_{\infty}+i \sqrt{B_{\infty}-\frac{1}{4} P_{\infty}}\right)^{-1} .
$$

In the numerical realization, the height $z_{\infty}$ is taken to be $430 \mathrm{~km}$ above which $U$ is constant in our model. Under the lower boundary condition $(z=0)$, we set

$$
G(0)=0 \text {. }
$$

This condition, according to (11), is equivalent to the condition of equality to the null function of $v_{z}$.

By solving initial value problem (10), (12) for $G$, we find values of the dispersion function that should meet the condition

$$
D\left(\omega, k_{x}\right)=G\left(0, \omega, k_{x}\right)=0 .
$$

Formally, Equation (14) can be solved by setting the first or the second argument of the dispersion function $D$ to be real. In the former case, we have modes attenuating (due to the non-Hermitian character of the problem) in horizontal propagation; and in the latter case, modes damping in time. In this paper, we analyze modes only with real values of frequency $\omega$. The vertical spatial structure of the mode for two dispersion values of $\omega$ and $k_{x}$ satisfying Equation (14) can be obtained by numerically solving the Cauchy problem for Equations (9) with the initial condition $v_{z}(0)=0, v_{z}^{\prime}(0)=1$ corresponding to the selection of boundary condition (13) for $G$.

\section{Boundary value problem with dissipation (BVPD)}

Since boundary conditions at the upper boundary for the waveguide modes are the same as for DSAS [Rudenko, Dmitrienko, 2015], it suffices to choose DSAS that meets the condition of equality to null vertical velocity on Earth's surface to solve the waveguide problem. Hence, the respective dispersion equation takes the form

$$
D\left(\omega, k_{x}\right)=v_{z}\left(0, \omega, k_{x}\right)=0 .
$$

It is complex due to penetration through a barrier and the presence of dissipation.

\subsection{Numerical calculations of characteristics of internal gravity waveguide modes}

The calculations of waveguide modes have first and foremost established that for the frequency range corresponding to TIDs in the chosen model atmosphere there is only one nodeless waveguide mode with $n=0$, as was shown by all algorithms described in 3.1 .

For a general analysis of propagation characteristics of the resulting waveguide mode, Figure 3 gives: 
- the dispersion dependence of horizontal wavelength on an oscillation period (the solid curve is similar for the BVPD and BVP problems; the dotted curve denotes MBSCQ);

- leaking wave characteristics formally referred to $z=z_{c}$ : total phase velocity (dashed curve); vertical group velocity (dash-dot); vertical wavelength (dash with three dots).

Figure 4 depicts characteristics of horizontal 0-mode attenuation: BVPD (solid curve), BVP (dashed curve), MBSCQ (dotted curve)

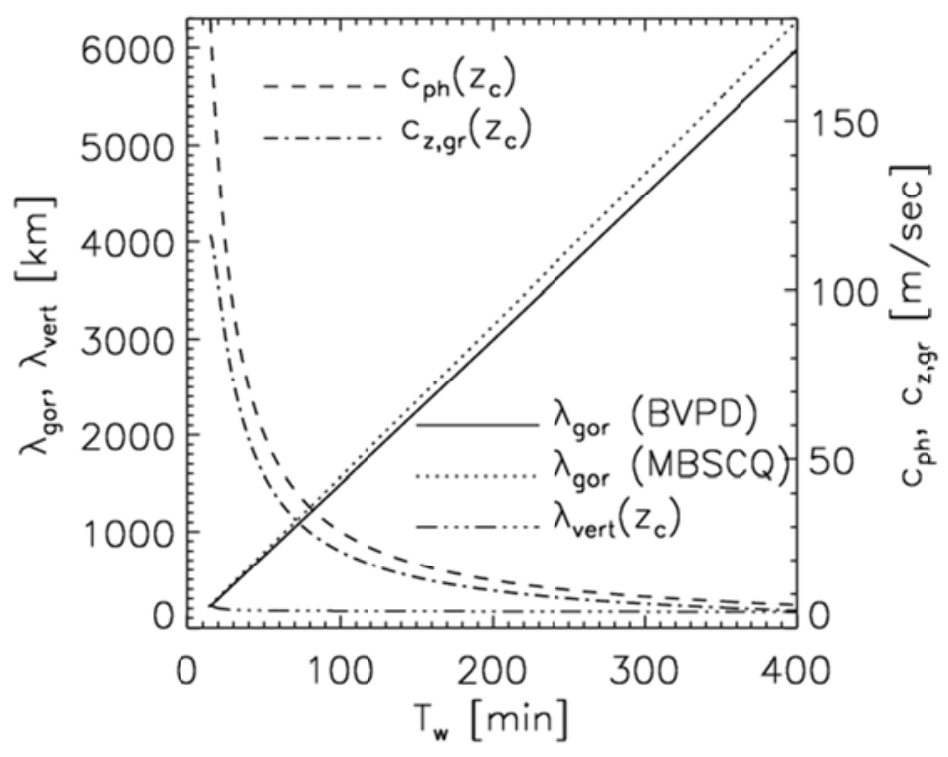

Figure 3. Waveguide characteristics of 0 mode: horizontal wavelength, BVPD=BVP (solid curve); horizontal wavelength, MBSCQ (dotted curve); total phase velocity of a runaway wave (dashed curve); vertical group velocity of a runaway wave (dash-dot); vertical wavelength of a runaway wave (dash with three dots)

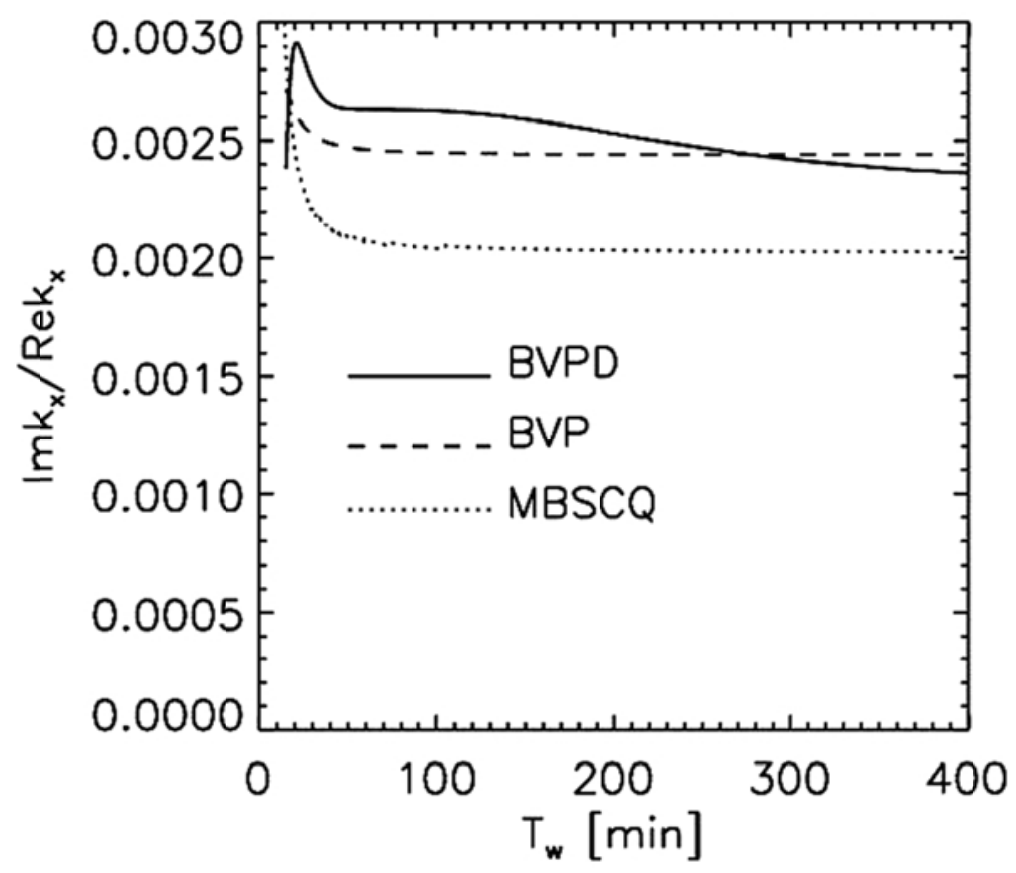

Figure 4. Horizontal 0-mode attenuation BVPD (solid curve), BVP (dashed curve), MBSCQ (dotted curve) 
Table. Comparison between theoretical and experimental results

\begin{tabular}{|c|c|c|c|c|}
\hline & $\begin{array}{c}\text { Elevation } \\
\left(T_{W}=82 \mathrm{~min}\right)\end{array}$ & $\begin{array}{c}\text { Elevation } \\
\left(T_{W}=182 \mathrm{~min}\right)\end{array}$ & $\begin{array}{c}\text { Velocity } \\
\left(T_{W}=82 \mathrm{~min}\right)\end{array}$ & $\begin{array}{c}\text { Velocity } \\
\left(T_{W}=182 \mathrm{~min}\right)\end{array}$ \\
\hline $\mathrm{P} 2$ & {$\left[-78^{\circ},-71^{\circ}\right]$} & {$\left[-86^{\circ},-731^{\circ}\right]$} & {$[18,28](\mathrm{m} / \mathrm{s})$} & {$[19.5,32](\mathrm{m} / \mathrm{s})$} \\
\hline Ours & $-82^{\circ}$ & $-86^{\circ}$ & $35(\mathrm{~m} / \mathrm{s})$ & $15.6(\mathrm{~m} / \mathrm{s})$ \\
\hline
\end{tabular}

Note the most crucial points:

- The model atmosphere in hand suggests the existence of only one mode. Since the chosen time of the model for the geographic location considered represents the most frequent facts of detection of ionospheric disturbances, we can assume that the conditions (anywhere) for two or more modes can either extremely rarely or never be realized.

- The three methods (BVPD, BVP, and MBSCQ) gave close values both for the horizontal velocity of waveguide mode propagation and for the horizontal attenuation. The horizontal attenuation is quite low for very-long-distance propagation of waveguide disturbances. The close agreement between the results of the exact methods with the result of the quasi-classical description formally inapplicable to the zero mode is a fairly interesting fact from a methodological point of view.

- Of particular interest is the property that seems to be specific only for IGW modes with penetration. The dependences presented in Figure 4 show a high Q-factor of waveguide oscillations at a rather slight decrease in the waveguide solution amplitude after passing through the opacity barrier $\left[z_{1}\right.$, $\left.z_{2}\right]$. The parameter $\sqrt{Q}$, found in (3), is 0.41 . For an ordinary acoustic waveguide mode, such a value of $\sqrt{Q}$ could cause a quite strong horizontal attenuation of around $0.41^{2}$. For IGW, the factor of the first term in (6) takes on a value of $\sim 30$ (for sound, $\sim 1$ ), providing a high Q-factor for modes of this type. From physical standpoint, this effect is assured by infinitesimal of the vertical group velocity of a leaking wave.

Also noteworthy is that the dispersion characteristic of zero mode we obtained reproduces very well the characteristic relation of horizontal scales with periods of the observed TIDs. Additionally, we have good agreement of our values of the total phase velocity in the upper atmosphere with the observed phase velocities of TIDs [Ratovsky et al., 2008; Medvedev et al., 2009]. These results are consistent with the waveguide concept of TID nature. We thoroughly compare properties of waveguide disturbances with the observed TID properties described in [Medvedev et al., 2013]. Medvedev et al. [Medvedev et al., 2013] examined a space-time structure, analyzing data on electron density profiles of two beams of the Irkutsk Incoherent Scatter Radar and digisonde.

Compare first the Elevation and Velocity values (given in Figure 1, [Medvedev et al., 2013]), obtained from a 12-hour window spectral analysis, with equivalent values of the angular characteristic $\operatorname{Atan} \frac{k_{\mathrm{vert}}\left(z_{c}\right)}{k_{\mathrm{hor}}\left(z_{c}\right)}$ and velocity $c_{\mathrm{ph}}\left(z_{c}\right)$ respectively. 
The values listed in Table are assessed because they contain $k_{\text {vert }}$ whose application conditions at heights of around $z_{c}$ are not fulfilled. Moreover, the presence of the theoretically disregarded real wind at the heights of interest can lead to a significant shift in observed characteristics relative to theoretical ones. It is sufficient for us that the theoretically predicted characteristics fit the observed ranges of these characteristics. Also interesting is to compare our values with the most probable Elevation, Velocity, and Wavelength values $\left(\approx \lambda_{\text {vert }}\right)$ presented in Figures 4-6 in [Medvedev et al., 2013]. Especially notable are left panels of these figures for daytime recordings according to the daytime model atmosphere adopted. Note that in the daytime recordings the time spectrum of signals is calculated in the limited time range from $\sim 1$ to $\sim 3 \mathrm{hr}$ with two local peaks in the vicinity of 3 and $1.5 \mathrm{hr}$ (Figure 2, [Medvedev et al., 2013]). The first peak is the highest with a narrow width; the second is broader with lower amplitude. Our Elevation and Wavelength values most closely fit the observations. The most probable Elevation value (Figure 4, [Medvedev et al., 2013]), equal to $-75^{\circ}$, is close to our values (see Table). The most likely Wavelength value of $175 \mathrm{~km}$ (Figure 6, [Medvedev et al., 2013]) is very similar to our value $\lambda_{\text {vert }}=192 \mathrm{~km}$ (Figure 3 ). The most probable Velocity value (Figure 5 , [Medvedev et al., 2013]), equal to $35 \mathrm{~m} / \mathrm{s}$, match the period $T_{W}=1.4 \mathrm{hr}$ in our plot in Figure 3.

This value of $T_{W}$ fits one of the spectral distribution peaks (Figure 2, [Medvedev et al., 2013]). Hence, we see that our theoretical description is supported by observations.

\subsection{The height structure of inner gravity waveguide mode}

After finding the waveguide dispersion relations, to determine the height structure of the waveguide solution we should calculate DSAS for two wave parameters $\omega$ and $k_{x}$ related by the dispersion characteristic. This procedure has been described in the BVPD solution scheme. In the BVP scheme, we can derive the dependence $v_{z}(z)$, using the $G\left(z, \omega, k_{x}\right)$ function found with the BVP procedure. To do this, it suffices to numerically integrate the first-order differential equation $v_{z}^{\prime}=v_{z} / G$ with the initial value $v_{z}\left(z_{\infty}\right)=1$. We employ the BVP result for the waveguide solution only to compare it with the solution according to our basic BVPD scheme. The comparison relies on vertical distributions of $v_{-}(z)=\left(\frac{\rho_{0}}{\rho_{0}(0)}\right)^{1 / 2} \frac{v_{z}}{c_{z}}$. Figure 5 compares values $\operatorname{Re} v_{-}$of BVPD and BVP solutions at a wave period $T_{W}=90 \mathrm{~min}$ that has respective eigenvalues of the horizontal wavenumber $k_{x}=\left(4.66233 \cdot 10^{-}\right.$

$\left.{ }^{3}+i 1.22515 \cdot 10^{-5}\right) \mathrm{km}^{-1}$ for BVP and $k_{x}=\left(4.66215 \cdot 10^{-3}+i 1.14273 \cdot 10^{-5}\right) \mathrm{km}^{-1}$ for BVPD. We see that both the solutions are very close to each other in the region $R^{\mathrm{III}}$ with small dissipation. Above them, the plots are, of course, essentially different. The BVP solution comes to wave asymptotics in a homogeneous medium, while DSAS (BVPD) attenuates under the action of wave dissipation.

Thus, Figure 5 clearly shows that the dissipationless wave description is valid only for a limited height range with the infinitesimal condition for $s$ being fulfilled. 


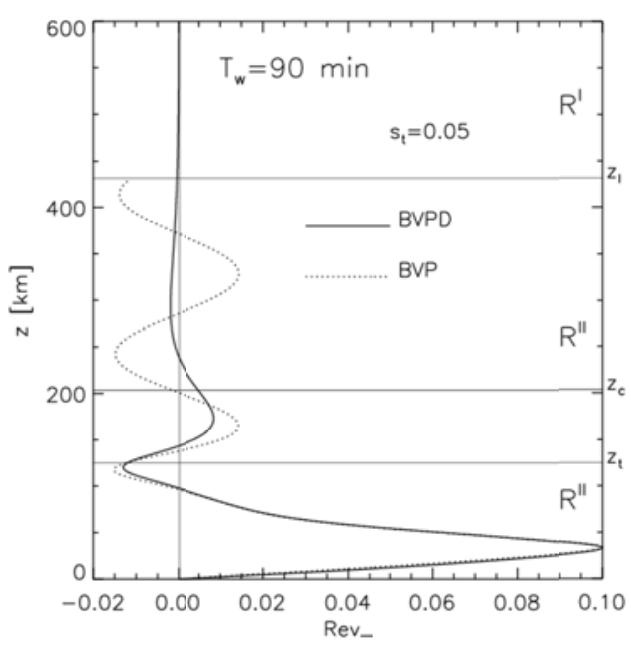

Figure 5. Comparison between DSAS and the dissipationless waveguide solution

To facilitate the graphical representation of BVPD, we employ two sets of vallues. To display the upper part of the waveguide solution, we use $\Theta, n, f, v, u$ determined by Equalities (17) taken from [Rudenko, Dmitrienko, 2015]. The lower part is represented by the following modifications of the values:

$$
\begin{aligned}
& \Theta^{-}=W \Theta, \\
& n^{-}=W n, \\
& f^{-}=W f, \\
& v^{-}=10 W v, \\
& u^{-}=W u .
\end{aligned}
$$

Here $W(z)=\left(\frac{\rho_{0}(z)}{\rho_{0}(200 \mathrm{~km})}\right)^{1 / 2}$. All the following representations of wave solutions are based on the normalization $\max \left(\sqrt{v_{x}^{2}+v_{z}^{2}}\right)=50 \mathrm{~m} / \mathrm{s}$.

Discuss the first example of the waveguide structure in Figure 6. This figure illustrates $z$ dependences of real and imaginary parts of all disturbance components ((17) from [Rudenko, Dmitrienko, 2015] and (16))

The top plots show the dependence of relative disturbance components. The bottom plots describe details of vertical structures that do not appear in top ones because the exponential factor. We see that the relative values are largest in the upper atmosphere; and absolute ones, in the lower atmosphere. On reaching a maximum, the relative values start decreasing due to dissipation. In the scale we presented, all values seem continuous at $z_{s}$. Indices of solution discontinuity for this case are rather low:

$$
\begin{gathered}
\delta_{[\Theta]}=\delta_{[\mathrm{T}]}=\delta_{[\Theta-]}=0.134, \\
\delta_{[n]}=\delta_{[Q]}=\delta_{[\mathrm{n}-]}=0.095 .
\end{gathered}
$$

Thus, we derive a solution that is qualitatively close to the solutions found by the isothermal model atmosphere (Section 5, [Rudenko, Dmitrienko, 2015]). Note that according to the calculations discontinuity indices are of the same order for all points of the dispersion curve. 
The $W(z)$ values on the bottom panels in Figure 6, the undisturbed temperature (Figure 1), pressure, density, and velocity of sound (Figure 7) enable us to calculate absolute values of disturbances at any height.

For an indirect test, we can compute a numerical parameter similar to the parameter of total vertical absorption $\eta$ from [Rudenko, Dmitrienko, 2015] ((43), [Rudenko, Dmitrienko, 2015]) for the isothermal model atmosphere. In the isothermal case, $\eta$ is equal to the ratio of the $T$ amplitude att $z_{c}$ to the amplitude of a dissipationless upward propagating wave at the same height. If, as in our case, the reflected wave fraction at $z_{c}$ is small, then, instead of dissipationless incident wave, we can take a complete combination of the incident wave with the reflected one. On this basis, we select $\eta_{\text {calc }}$ similar to $\eta$ for an inhomogeneous atmosphere. To calculate this value, we solve the Cauchy problem for the system of equations (2) (9) (10) from [Rudenko, Dmitrienko, 2015] from bottom to top to $z_{c}$, using $p$ and $v$ from DSAS on Earth's surface as boundary values. We compute $\eta_{\text {calc }}$ as a ratio of the modulus $T$ at $z_{c}$ to the modulus $T$ of the dissipationless wave solution derived at this height. An example of such a calculation is given in Figure 8. This figure once more confirms that the dissipation and dissipationless solutions begin to differ markedly at $z_{s}$. It can be seen that at $z_{c}$ these solutions are essentially different.

We compare $\eta_{\text {calc }}$ with $\eta$ estimated for the isothermal mode in which the temperature of a medium is chosen equal to $T_{0}$ of the nonisothermal model at $z_{c}$. The result of the comparison between these values throughout the 0 -mode spectrum is presented in Figure 9.
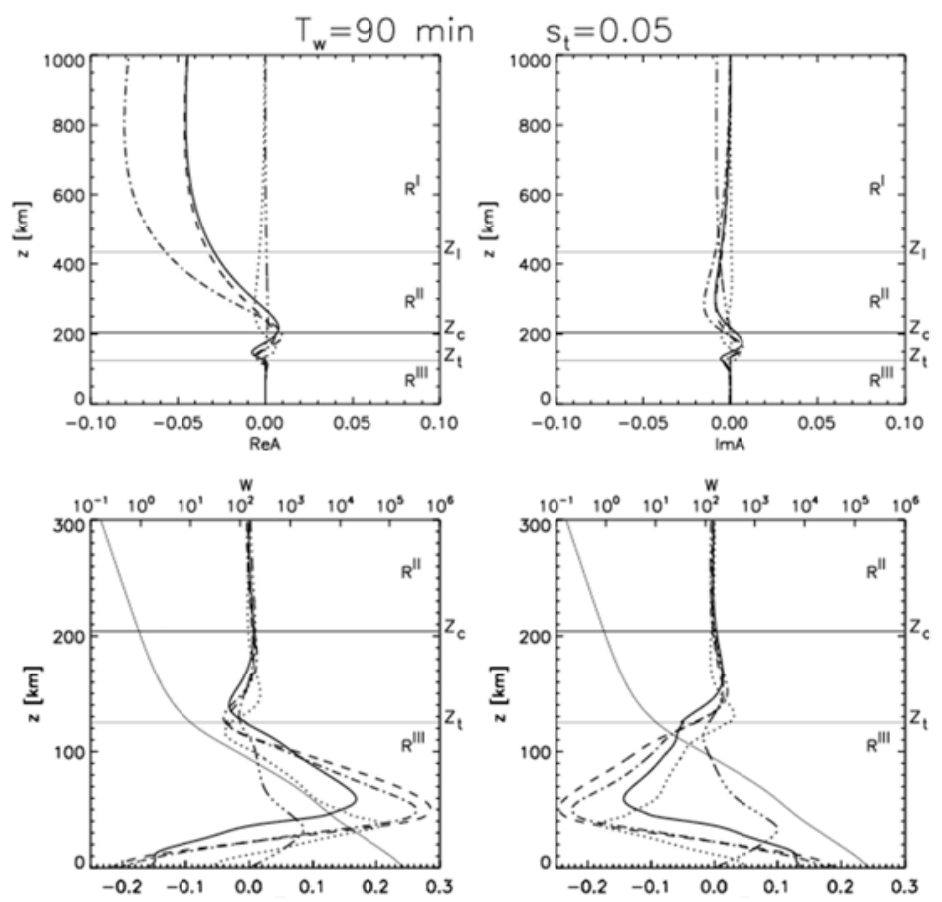

Figure 6. Full structure of the waveguide solution for $T_{W}=90 \mathrm{~min}$. The correspondence between lines and wave values is the same as in Figure 6 from [Rudenko, Dmitrienko, 2015] (the dotted line marks $\Theta$; the dash with three dots, $v$; the solid line, $n$; the dashed line, $f$; the dashed-dot line, $u$ ). The thin solid line depicts the vertical distribution of $W(z)$ 

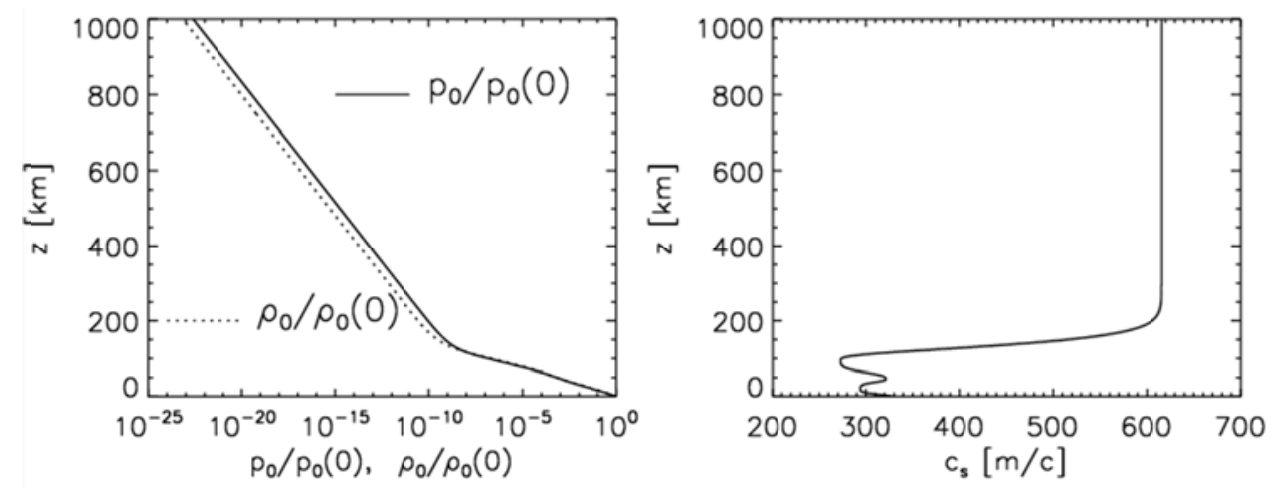

Figure 7. Height dependences of undisturbed pressure, density, and velocity of sound

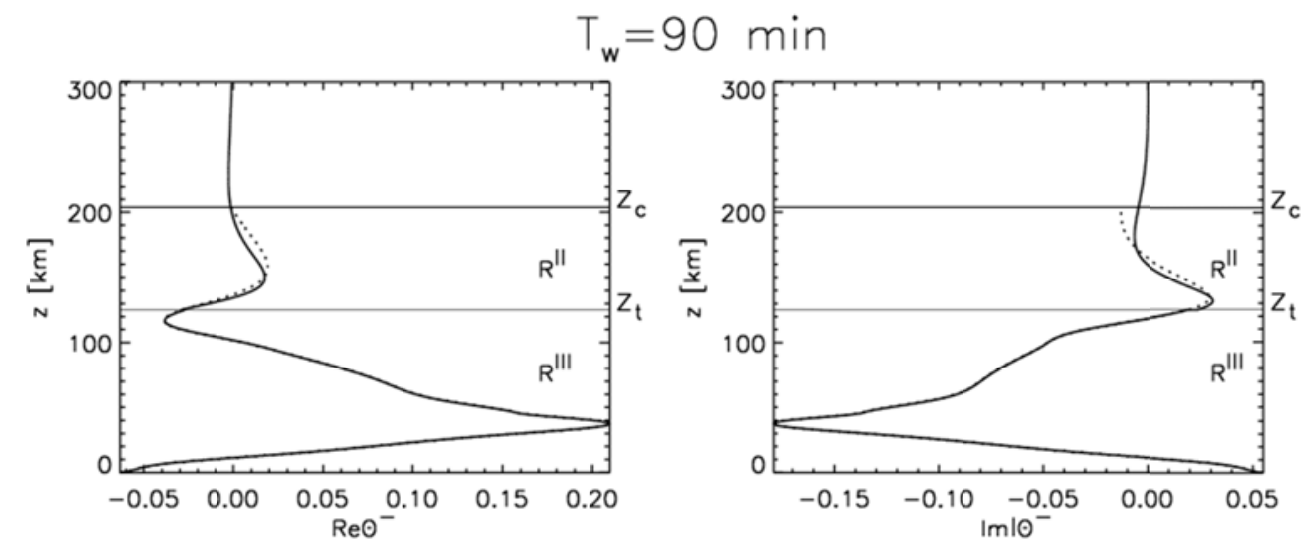

Figure 8. Modulus of temperature disturbance in the waveguide solutions with and without dissipation (solid and dotted curves respectively)

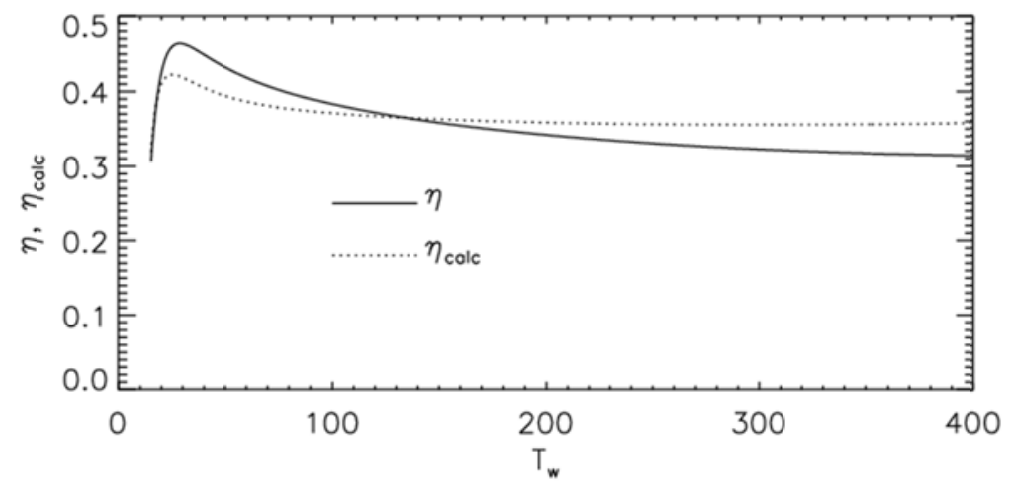

Figure 9. Comparison between $\eta_{\text {calc }}$ and $\eta$ values

The last figure indicates that the order of the index of the total vertical absorption in the wave solution on the interval $z=\left[-\infty, z_{c}\right] \eta=\left(-\infty, z_{c}\right) \approx \eta\left(z_{s}, z_{c}\right)$. The closeness between $\eta_{\text {calc }}$ and isothermal $\eta$ is a qualitative confirmation of DSAS validity. Note that, with $\eta_{\text {calc }}$ and $\eta$ being determined through complex values of their defining quantities, the complex $\eta_{\text {calc }}$ and $\eta$ values are close to each other with almost the same accuracy. We do not present the calculation results of $\eta_{\text {calc }}$ and $\eta$. 

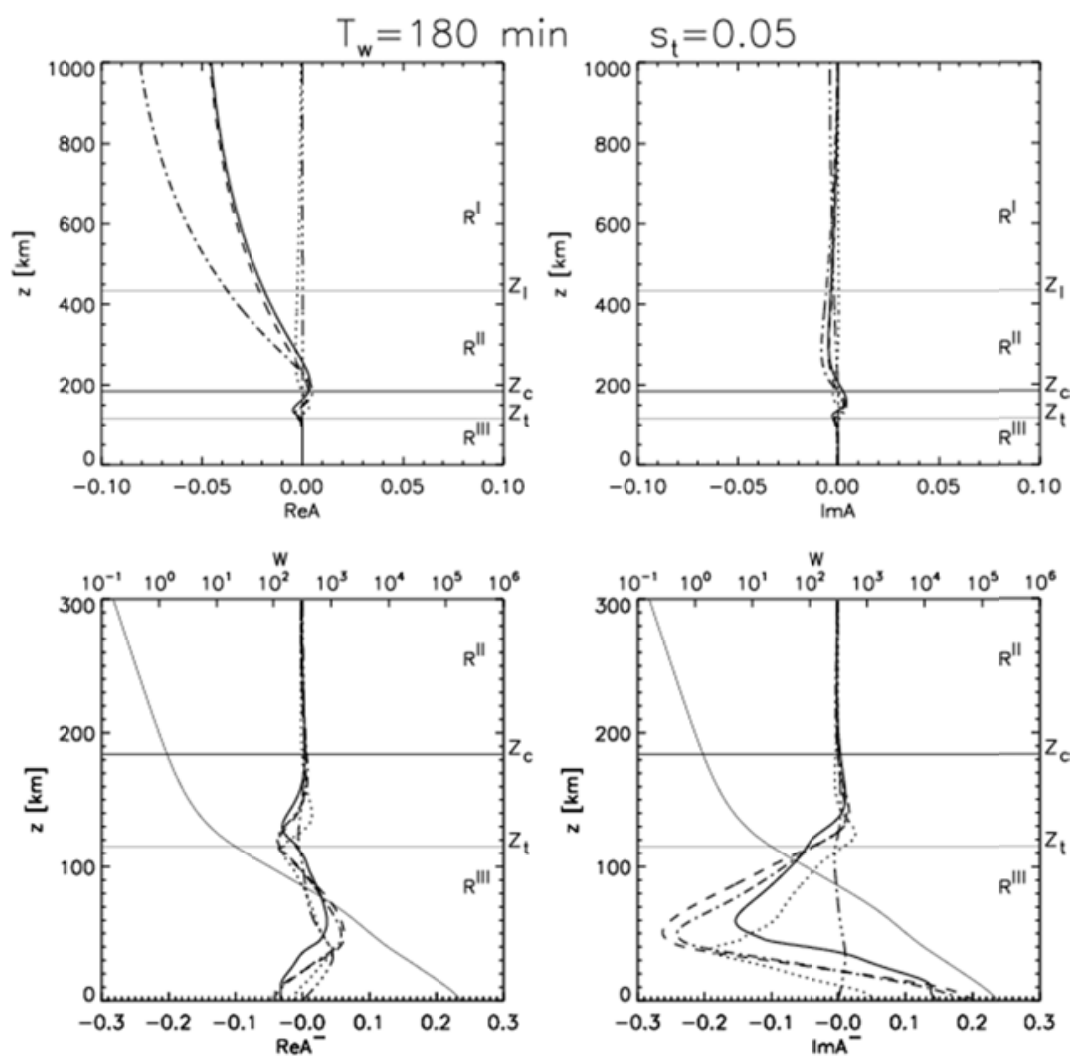

Figure 10. Full structure of the waveguide solution for $T_{W}=180 \mathrm{~min}$

To more fully represent the waveguide mode, we also give Figures $10-12$ for $T_{W W}=180 \mathrm{~min}$ and for boundary points of the spectral range $T_{W}=15 \mathrm{~min}$ and $T_{W}=400 \mathrm{~min}$.

Note that in the longwave spectrum range (Figures 10,12) we do not see that some relative values stop increasing in the calculated height interval, but it is clear that they attenuate outside it because continuations of numerical solutions are attenuated analytical solutions.

\section{CONCLUSION}

We have studied IGW waveguide modes that feature sub-barrier penetration into the upper atmosphere ensuring their penetration to ionospheric heights. Because of the exponential decrease in atmospheric density, relative values of an upward propagating disturbance exponentially increase. As a result, trapped waves manifest themselves at large heights and cause «visible» ionospheric traveling disturbances over long distances. For the model atmosphere adopted here, we have revealed that with a given frequency there is only one basic (nodeless) mode. We calculated a dispersion curve for the waveguide mode - a complex horizontal number as a function of real frequency. 

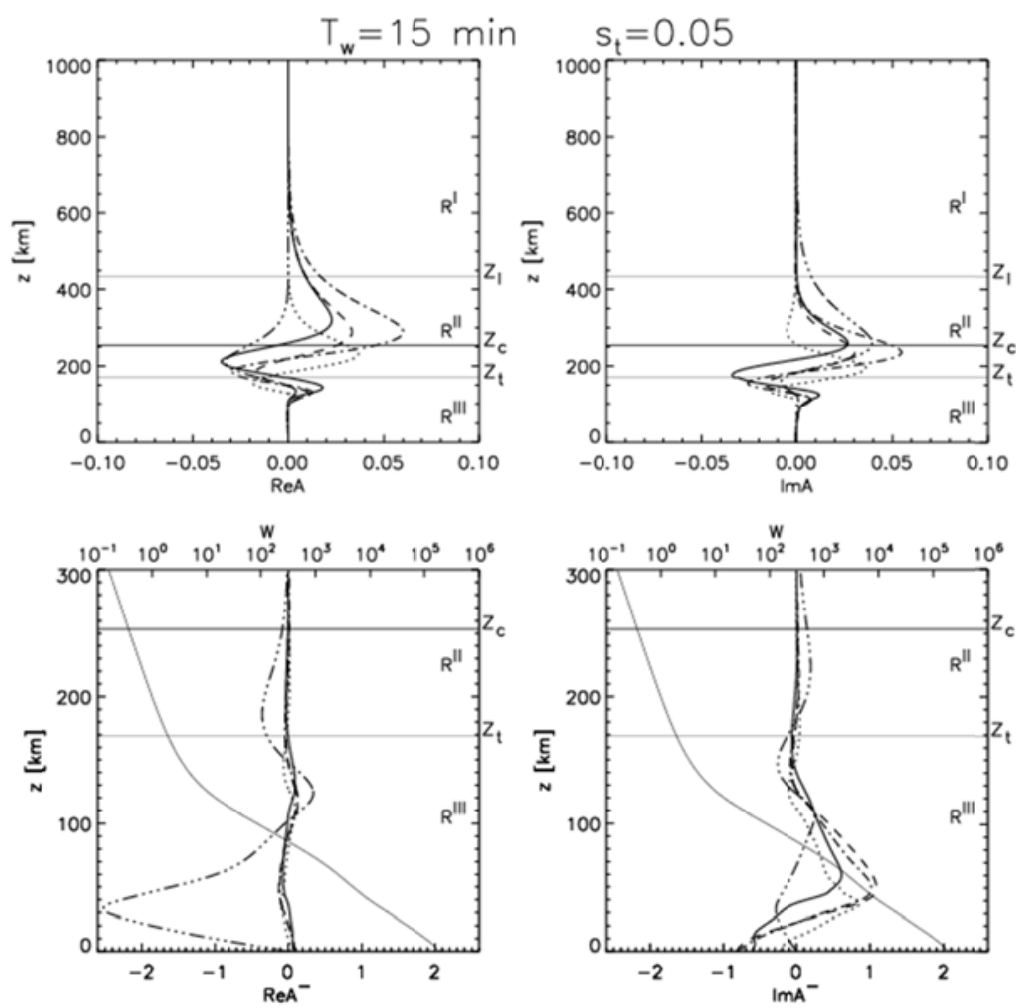

Figure 11. Full structure of the waveguide solution for $T_{W}=15 \mathrm{~min}$
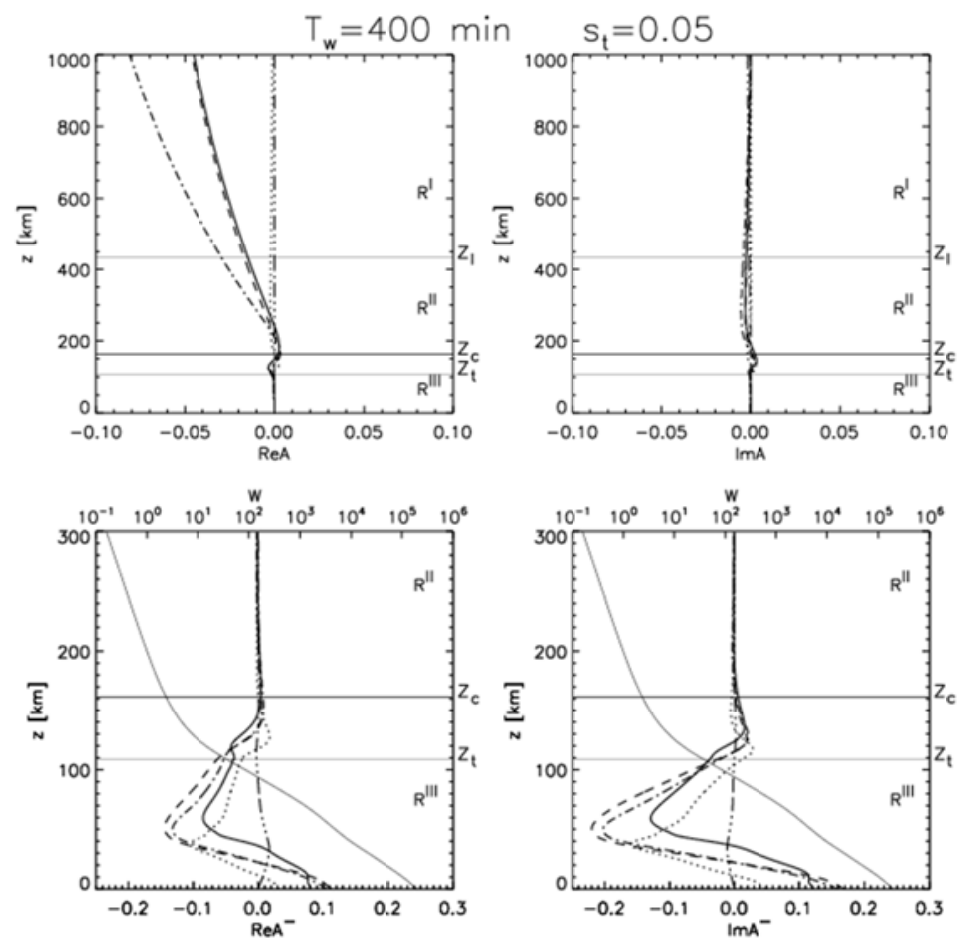

Figure 12. Full structure of the waveguide solution for $T_{W}=400 \mathrm{~min}$

We have first presented the vertical IGW-mode structure along with its part escaping from the waveguide in their entirety at all heights, with full regard to dissipation. We managed to do this by adopting the method for finding a dissipative solution over a source [Rudenko, Dmitrienko, 2015]. 
In this study, in addition to the dissipative solution, we have explored possibilities of dissipationless description. We showed that, despite its being inapplicable to the upper atmosphere, the dissipationless description appears to work fairly well in the lower atmosphere. It enables us to estimate the number of modes and derive testing values for numerical calculation of eigenvalues; the most convenient for these objectives is the WKB method. It is interesting to note the WKB description provides correct results despite its formal invalidity; yet there is good agreement between dispersion WKB characteristics with exact ones.

We have demonstrated that the method we proposed does not give any significant errors comparable to errors in observational data. The specific calculations we made have an independent error related to the statistical character of the NRLMSISE model. This is the most significant factor of possible differences, obtained with this model, from observed values. Thus, we should generally compare our results with observable statistical characteristics. Therefore, the comparisons with experimental data we performed look sufficiently convincing. Using the dispersion relations and characteristics of wave propagation of runaway disturbance, we have obtained very good agreement with basic characteristics of observed TIDs: the ratio between horizontal scales and wave periods, the ability to propagate to many thousands of kilometers without significant attenuation; the reverse direction of vertical phase velocity; small values of vertical phase velocity, the specific slope of the phase front.

We are grateful to Medvedev A.V. and Ratovsky K.G. for the useful contribution to our study.

\section{REFERENCES}

Afraimovich E.L., Kosogorov E.A., Lesyuta O.S., Ushakov I.I., Yakovets A.F. Geomagnetic control of the spectrum of traveling ionospheric disturbances based on data from a global GPS network. Ann. Geophys. 2001, vol. 19, iss. 7, pp. 723-731. DOI: 10.5194/angeo-19-723-2001.

Akhmedov P.P., Kunitsyn V.E. Modeling of ionospheric disturbances caused by earthquakes and explosions. Geomagnetizm i Aeronomiya [Geomagnetism and Aeronomy] 2004, vol. 44, no. 1, pp. 1-8 (in Russian).

Francis S.H. Acoustic-gravity modes and large-scale traveling ionospheric disturbances of a realistic, dissipative atmosphere. J. Geophys. Res. 1973a, vol. 78, p. 2278.

Francis S.H. Lower-atmospheric gravity modes and their relation to medium scale traveling ionospheric disturbances. J. Geophys. Res. 1973b, vol. 78, pp. 8289-8295.

Heale C.J., Snively J.B., Hickey M.P., Ali C.J. Thermospheric dissipation of upward propagating gravity wave packets. J. Geophys. Res.: Space Physics. 2014, vol. 119, iss. 5, pp. 3857-3872. DOI: 10.1002/2013JA019387.

Hedlin Michael A.H., Drob Douglas P. Statistical characterization of atmospheric gravity waves by seismoacoustic observations. J. Geophys. Res. Atmos. 2014, vol. 119, iss. 9, pp. 5345-5363. DOI: 10.1002/2013JD021304.

Hines C.O. Internal atmospheric gravity waves at ionospheric heights. Can. J. Phys. 1960, vol. 38, pp. 1441-1481.

Hocke K., Schlegel K. A review of atmospheric gravity waves and travelling ionospheric disturbances: 19821995. Ann. Geophys. 1996, vol. 14, pp. 917-940.

Hunsucker R.V. Atmospheric propagation of atmospheric gravity waves: A review. Rev. Geophys. Space Phys. 1982, vol. 20, pp. 293-315. 
Idrus Intan Izafina, Abdullah Mardina, Hasbi Alina Marie, Husin Asnawi, Yatim Baharuddin. Large-scale traveling ionospheric disturbances observed using GPS receivers over high-latitude and equatorial regions. J. Atmos. Solar-Terr. Phys. 2013, vol. 102, pp. 321-328. DOI: 10.1016/j.jastp.2013.06.014.

Kirchengast G., Hocke K., Schlegel K. Gravity waves determined by modelling of travelling ionospheric disturbances in incoherent scatter radar measurements. Radio Science. 1995, vol. 30, pp. 1551-1567.

Ma S.Y., Schlegel K., Xu J.S. Case studies of the propagation characteristics of auroral TIDs with EISCAT CP2 data using maximum entropy cross-spectral analysis. Ann. Geophys. 1998, vol. 16, no. 2, pp. 161-167.

Medvedev A.V., Ratovsky K.G., Tolstikov M.V., Kushnarev D.S. Method for studying space-time structure of wave disturbances in the ionosphere. Geomagnetizm i Aeronomiya [Geomagnetism and Aeronomy] 2009, vol. 49, no. 6, pp. 812-823 (in Russian).

Medvedev A.V., Ratovsky K.G., Tolstikov M.V., Alsatkin S.S., Scherbakov A.A.. Studying of the spatialtemporal structure of wavelike ionospheric disturbances on the base of Irkutsk incoherent scatter radar and digisonde data. J. Atmos. Solar-Terr. Phys. 2013, vol. 105, pp. 350-357.

Oliver W.L., Fukao S., Sato T., Tsuda T., Kato S., Kimura I., Ito A., Saryou T., Araki T. Ionospheric incoherent scatter measurements with the middle and upper atmosphere radar: Observations during the large magnetic storm of February 6-8. J. Geophys. Res. 1988, vol. 93, no. A12, pp. 14649-14655.

Ratovsky K.G., Medvedev A.V., Tolstikov M.V., Kushnarev D.S. Case studies of height structure of TID propagation characteristics using cross-correlation analysis of incoherent scatter radar and DPS-4 ionosonde data. Adv. Space Res. 2008, vol. 41, pp. 1453-1457.

Rudenko G.V., Dmitrienko I.S. Wave above the source in the heat-conducting atmosphere. Solnechno-Zemnaya Fizika [Solar-Terrestrial Physics]. 2015, vol. 1, iss. 4, pp. 11-29 (in Russian).

Shibata T., Okuzawa T. Horizontal velocity dispersion of medium-scale travelling ionospheric disturbances in the F-region. J. Atmos. Terr. Phys. 1983, vol. 45, pp. 149-159.

Vadas S.L., Nicolls M.J. Using PFISR measurements and gravity wave dissipative theory to determine the neutral, background thermospheric winds. Geophys. Res. Lett. 2008, vol. 35, iss. 2, CiteID L02105. URL: http://dx.doi.org/10.1029/2007GL 031522 (accessed August 15, 2015).

Vadas S.L., Nicolls M.J. The phases and amplitudes of gravity waves propagating and dissipating in the thermosphere: Theory. J. Geophys. Res. 2012, vol. 117, iss. A5, CiteID A05322. DOI: 10.1029/2011JA017426.

Vadas Sharon L., Liu Han-li. Generation of large-scale gravity waves and neutral winds in the thermosphere from the dissipation of convectively generated gravity waves. J. Geophys. Res. 2009, vol. 114, iss. A10, CiteID A10310. DOI: 10.1029/2009 JA014108.

Williams P.J.S., Virdi T.S., Lewis R.V., Lester M., Rodger A.S., Freeman K.S.C. Worldwide atmospheric gravity wave study in the European sector 1985-1990. J. Atmos. Solar-Terr. Phys. 1993, vol. 55, no. 4-5, pp. 683-696. 